\title{
Effects of Exercise Training Interventions on Executive Function in Older Adults: A Systematic Review and Meta-Analysis
}

\author{
Feng-Tzu Chen ${ }^{1} \cdot$ Jennifer L. Etnier ${ }^{2} \cdot$ Kuei-Hui Chan ${ }^{3} \cdot$ Ping-Kun Chiu ${ }^{3} \cdot$ Tsung-Ming Hung $^{4,5} \cdot$ Yu-Kai Chang ${ }^{4,5}$
}

Published online: 23 May 2020

(c) The Author(s) 2020

\begin{abstract}
Background Chronic exercise training has been shown be to positively associated with executive function (EF) in older adults. However, whether the exercise training effect on EF is affected by moderators including the specific sub-domain of $\mathrm{EF}$, exercise prescription variables, and sample characteristics remains unknown.

Objectives This systematic and meta-analytic review of randomized controlled trials (RCTs) investigated the effects of exercise training on EF in older adults and explored potential moderators underlying the effects of exercise training on EF. Methods In accordance with the PRISMA guidelines, the electronic databases MEDLINE (PubMed) and EMBASE (Scopus) were searched from January 2003 to November 2019. All studies identified for inclusion were peer-reviewed and published in English. To be included, studies had to report findings from older ( $>55$ years old), cognitively normal adults or adults with mild cognitive impairment (MCI) randomized to an exercise training or a control group. The risk of bias in each study was appraised using the Cochrane risk-of-bias tool. Fixed-effects models were used to compare the effects of exercise training and control conditions on EF assessed at baseline and post-intervention. In addition, subgroup analyses were performed for three moderators (i.e., the specific sub-domain of EF, exercise prescription variables, and sample characteristics).

Results Thirty-three RCTs were included. Overall, exercise training was associated with a significant small improvement in $\mathrm{EF}[Q(106)=260.09$, Hedges' $g=0.21 ; p<0.01]$. The EF sub-domain moderator was not significant $[Q(2)=4.33, p>0.05]$, showing that the EF improvement in response to exercise is evident for measures of inhibition, updating, and shifting. Regarding exercise prescription variables, results were significantly moderated by frequency of exercise training $[Q(1)=10.86$, $p<0.05$ ], revealing that effect sizes (ESs) were larger for moderate frequency $(g=0.31)$ as compared to low frequency exercise $(g=0.15)$. The results also showed type of exercise training moderated the ESs $[Q(4)=26.18, p<0.05]$, revealing that ESs were largest for other forms of exercise $(g=0.44)$, followed by Tai Chi and yoga $(g=0.38)$, resistance exercise $(g=0.22)$, aerobic exercise $(g=0.14)$, and combined exercise $(g=0.10)$. In addition, The results showed moderated length of training the ESs $[Q(2)=16.64, p<0.05]$, revealing that ESs were largest for short length $(g=0.32)$, followed by mid length $(g=0.26)$ and long length $(g=0.09)$. No significant difference in effects was observed as a function of exercise intensity $[Q(1)=2.87 p>0.05]$ and session time $[Q(2)=0.21, p>0.05]$. Regarding sample characteristics, the results were significantly moderated by age $[Q(2)=20.64, p<0.05]$, with significant benefits for young-old (55-65 years old) $(g=0.30)$ and mid-old (66-75 years old) $(g=0.25)$, but no effect on EF for old-old (more than 75 years old). The results were also significantly moderated by physical fitness levels $[Q(1)=10.80, p<0.05]$, revealing that ESs were larger for sedentary participants $(g=0.33)$ as compared to physically fit participants $(g=0.16)$. In addition, results were also significantly moderated by cognitive status $[Q(1)=11.44, p<0.05]$, revealing that ESs were larger for participants with cognitively normal $(g=0.26)$ as compared to those with mild cognitive impairment $(g=0.08)$. No significant differences in effects were observed as a function of $\operatorname{sex}[Q(2)=5.38, p>0.05]$.
\end{abstract}

Conclusions Exercise training showed a small beneficial effect on EF in older adults and the magnitude of the effect was different across some moderators.

Extended author information available on the last page of the article 


\section{Key Points}

Exercise training improves multiple domains of executive function (EF), including inhibition, updating, and shifting.

Exercise training leads to improved EF regardless of frequency, intensity, type, session time, length, and sex for all age groups except those over 75 years of age.

Exercise training with a frequency of 3-4 times per week, with vigorous intensity, and other forms of exercise/Tai Chi and yoga, as well as a length of training period from 1 to 3 months, produces larger beneficial effects on EF.

Young-old (55-65 years old) and mid-old (66-75 years old) individuals with intact cognitive abilities, particularly those who were initially sedentary, experience benefits on $\mathrm{EF}$ from exercise training interventions.

\section{Introduction}

Accumulating evidence suggests that chronic exercise is an important behavior for preventing cognitive decline and impairment in older populations. A positive association between physical activity and cognitive function has been observed in cross-sectional and longitudinal observational studies [1-4]. Further, the positive effects of chronic exercise training interventions on cognitive function have also been described in narrative reviews [5-9], and systematic and meta-analytic reviews of longitudinal studies have revealed that older adults engaged in exercise training interventions are protected against cognitive decline [10,11].

When considering the specificity of the beneficial effects of exercise on cognition, executive function $(\mathrm{EF})$ is observed to benefit from exercise training interventions as reported in previous meta-analyses [12-14]. In 2003, Colcombe and Kramer [12] conducted a seminal meta-analysis concluding that there was a strong relationship between exercise training and EF. At that time, EF was viewed as a broad construct in the exercise and cognition literature. However, since that time, the operationalization of EF tasks has become more specific and the EF tasks more diversified. EF encompasses basic and underlying cognitive functions for purposeful and goal-directed behavior [15] and EF is related to the neural activity of the prefrontal cortex [16-18]. Chronic exercise has been shown to be associated with activation of the prefrontal cortex [3, 19, 20], and exercise training has been shown to facilitate activation of the prefrontal cortex [21, 22]. EF is not a unitary construct but rather can be divided into (1) core EFs (i.e. inhibition, updating/working memory, switching), and (2) higher-level EFs (i.e. planning/problem solving) [23, 24]. While exercise may benefit these domains differentially, the question has been relatively unexplored in the older population. In addition, although previous narrative and meta-analytic reviews have demonstrated the effect of exercise training on EF broadly, the strength of this evidence is under debate $[25,26]$. Importantly, some researchers argue that the evidence for EF benefits from exercise is negligible [25], while others argue that the findings are consistent and positive [26]. Recent meta-analyses support an overall small positive effect of exercise on EF performance by older adults $[13,14]$ and indicate that the effect is influenced by some aspects of cognition (i.e., sub-domains of memory and $\mathrm{EF}$ ) [11], but there is no review to date which has explored the extent to which exercise-induced benefits differ across specific sub-domains of EF as currently explored in the exercise and cognition literature.

In addition, previous meta-analyses have also been hampered by experimental and methodological shortcomings. For instance, the American College of Sports Medicine (ACSM) has suggested that principles of exercise prescription aimed at improving health and physical fitness should be guided by the FITT (Frequency, Intensity, Time, Type) principle with specific recommendations made regarding frequency (how many days per week?), intensity (how hard is the exercise?), time (how long does each session last?) and type (what kind of exercise is being performed) [27]; however, previous meta-analyses associated with exercise training interventions and EF have provided relatively little information about these exercise prescription variables.

Last but not least, the effects of exercise training interventions appear to vary according to the characteristics of the sample in question, suggesting that personal demographics might influence the effects of exercise on EF [28-30]. Although two recent meta-analyses reported non-significant differences in effect sizes (ESs) for samples with or without mild cognitive impairment (MCI) [13, 14], other demographic variables (e.g., age, sex, and physical fitness) have not typically been considered as moderators. As such, one way to extend the existing knowledge regarding exercise training interventions and $\mathrm{EF}$ in older adults is to conduct a comprehensive meta-analysis in order to broadly examine additional moderators related to the sample characteristics.

Taken together, the present meta-analytic review is primarily aimed at examining the effects of exercise training interventions on EF in older adults who are either cognitively intact or have MCI. In addition, we considered whether these effects were influenced by moderators relative to three main topics: (1) determining whether exercise training interventions have general or selective effects on specific sub-domains of EF; (2) examining if EF is influenced by specific aspects of the prescribed exercise training interventions; and (3) examining if sample characteristics 
influence the effects of exercise training interventions on $\mathrm{EF}$ for older populations.

\section{Methods}

The review's protocol followed the Preferred Reporting Items for Systematic Review and Meta-Analysis (PRISMA). The meta-analysis was performed following the statement of the PRISMA guidelines [31] and Cochrane Collaboration handbook [32] in order to provide comprehensive and transparent reporting of methods and results.

\subsection{Search Strategy}

Electronic article searches were conducted for the period between January 2003 and November 2019 in MEDLINE (PubMed) and EMBASE (Scopus) databases. The search terms in this review were a combination of exercise intervention terms ("exercise" OR "physical activity" OR "aerobic" OR "fitness" OR "cardio" OR "VO 2 " OR "Tai Chi" OR "yoga" OR "resistance exercise" OR "weight training"), AND cognitive performance terms ("cognition" OR "cognitive function" OR "cognitive performance" OR "executive function" OR "executive control" OR "inhibition" OR "updating" OR "working memory" OR "switching" OR "planning"), AND aging population terms ("aging" OR "older" OR "55 years old"), AND selected terms regarding experimental designs ("randomized controlled trial" OR "control clinical trial" OR "randomized clinical trial"). We further searched for additional relevant articles in Google Scholar and identified potential studies for inclusion from previous meta-analyses.

\subsection{Eligibility Criteria}

Studies were included if they met the following criteria: (1) full-length, peer-reviewed study describing a randomized controlled trial (RCT) with human subjects exploring the effects of exercise training interventions on cognition and published in English; (2) participants were men or women aged 55 years and older with normal cognition or diagnosed with MCI; (3) any type of supervised exercise intervention with planned and structured physical activity with the intention of increasing or maintaining physical fitness. Of note, studies that employed one or combined two or more types of exercise modalities as an intervention were included; however, studies involving exercise training interventions combined with other non-exercise activities (e.g. cognitive training, drugs, and video games) and interventions that included unsupervised training sessions were not included; (4) studies that included participants who engaged in no contact, no treatment, waiting list, sham exercise, and alternative active treatments for the comparison condition; and (5) the EF tests had to be administered both at baseline and post-intervention.

\subsection{Study Selection and Data Extraction}

Two independent reviewers screened the titles and abstracts of all identified articles. Abstracts that matched the inclusion criteria were retrieved as full-text articles. Subsequently, full-text articles were reviewed by the two same reviewers. If there were any disagreements, a third reviewer was consulted until a consensus was achieved.

Two independent reviewers extracted relevant data: study identifiers (e.g. author name, year of publication) and study sample size, EF sub-domains (inhibition, updating/working memory, switching, and planning), exercise prescriptions (frequency, intensity, type, session time, and length), and participants' characteristics (age, sex, physical fitness level, and cognitive status). If relevant information was not provided in a given study, the first author contacted the authors of the study and made up to three requests for the data by email.

EF domains were generally coded based on four subdomains: inhibition, updating/working memory, switching, and planning $[23,24]$ and the specific EF task was also coded (Table 1). EF outcomes were recorded at the baseline and post-intervention time points. For those studies reporting follow-up outcomes, we chose the first time point following the exercise training intervention as the post-intervention time point.

Exercise prescription variables were recorded based upon exercise frequency, intensity, type, session time, and length. Specifically, the exercise frequency was coded as $\leq 2$ times/week, 3-4 times/week, or 5-7 times/week as has been done in a previous meta-analysis [14]. The exercise type was coded as aerobic exercise, resistance exercise, Tai Chi and yoga, combined exercise (i.e. the combination of two or more types of training), or other forms of exercise (e.g., dance, coordination exercises). The exercise intensity was coded as moderate (3.00-6.00 METs; $11-13$ on the rating of perceived exertion [RPE] of Borg scale; 40-60\% $\left.\mathrm{HRR} / V_{\mathrm{O}_{2 \max }} ; 55-70 \% \mathrm{HR}_{\max } ; 50-70 \% 1-\mathrm{RM}\right)$, or vigorous (6.01-9.00 METs; $14-16$ on the RPE of Borg scale; $61-85 \%$ $\mathrm{HRR} / V \mathrm{O}_{2 \max } ; 71-90 \% \mathrm{HR}_{\max } ; 71-84 \%$ 1-RM) based upon recommendations for exercise intensity terminology [33]. Session time was coded as short (less than $45 \mathrm{~min}$ ), moderate (45-60 min), or long (over $60 \mathrm{~min}$ ) [14]. The exercise length was coded as short (1-3 months), medium (4-6 months), or long (over 6 months) [12]. Sample characteristics such as age (young-old/55-65 years; mid-old/66-75 years; oldold/76-85 years) and sex (male; female; both) were coded [12]. We further added variables for physical fitness level (sedentary; fit) and cognitive status [normal; $\mathrm{MCI}$ ], and the 
Table 1 Classification of executive function assessments

\begin{tabular}{llll}
\hline Executive function & & & \\
\hline Inhibition & Updating/working memory & Switching & Planning \\
\hline Stroop Color-Word & N-back (2 back) & Task switching task (switch, local & Tower of London task \\
Stroop-Interference score & Spatial working memory (three & switch cost, global switch cost) & Six elements test \\
The California Older Adult Stroop & item) & Trail Making Test (part B or part & Greenwich test \\
test-interference & WCST (number of categories & B-part A) & Multiple errands test \\
Auditory Stroop test & completed) & Shifting-naming & Hotel test \\
Hayling sentence completion test & Digit Span task (backward or back- & Color trail test & Naturalistic action test \\
Eriksen flanker task-incongruent & ward-forward) & Spatial switching & RIPA Problem Solving \\
Go-No-Go Test & WAIS-letter number sequencing & Picture switching & Delay discounting task \\
Stop signal Task & Verbal fluency-FAS form (number & Arrow switching & The Iowa gambling task \\
The random number generation task & of words) & Attentive Matrices Test & Cambridge gambling task \\
& Rey-Osterrieth complex figure test & D-KEFS verbal fluency (category, & Raven's progressive matrices \\
& (delay) & switching) & \\
& List sorting task & The digit-letter task & \\
& Auditory verbal learning task (ver- & The plus-minus task & \\
& bal fluency) & Dimension-switching task & \\
& COWAT-verbal fluency & & \\
& Running memory task (total recall) & & \\
& Spatial running span task & &
\end{tabular}

WCST Wisconsin card sorting task, WAIS Wechsler adult intelligence scale, COWAT Control oral word association test, D-KEFS Delis-Kaplan executive function system, RIPA Ross information processing assessment

coding of these two variables was determined by descriptions provided in each article.

\subsection{Risk of Bias Assessment}

Two reviewers independently judged the risk of bias in each study using the Cochrane risk-of-bias tool [32], which was recommended in the existing literature related to exercise and cognition $[34,35]$ and also conforms to the guidelines of PRISMA [31]. For RCTs, the Cochrane Collaboration Guideline specifies six domains, including sequence generation, allocation concealment, blinding of participants, blinding of assessors, incomplete outcome data, and selective outcome reporting. The potential risk of bias under each domain was evaluated as "low risk", "high risk", or "unclear". Any disagreements were discussed with a third reviewer until a final decision was achieved.

\subsection{Statistical Analysis}

Version 2.0 of the comprehensive meta-analysis (CMA) software (Englewood, NJ) was utilized for the overall analysis of ES and the subgroup analyses of ES, with statistical significance being defined as a two-sided $p$ value of $<0.05$.

CMA software was used to compute Hedges' $g$. The format for this calculation was [(Ex-post) $-($ Ex-pre $)]-[($ Conpost $)-($ Con-pre $)] /$ pooled SD-pre. The ESs were aggregated to calculate an overall ES. A positive ES indicates that the benefits for the exercise group exceed the benefits for the control group and a negative ES represents a deterioration in the performance of the experimental group (that is, those engaged in exercise training interventions) that was larger than the deterioration experienced by the control group or that the control group improved more than the experimental group. According to the criteria of Cohen [36], the interpretation of the magnitude of the effect was such that $0.20-0.49$ was interpreted as small, $0.50-0.79$ was interpreted as moderate, and more than 0.80 was interpreted as large. Higgins $I^{2}$ values were used to assess statistical heterogeneity with possible values ranging from 0 to $100 \%(1-49 \%=$ low, $50-74 \%=$ moderate, $75-100 \%=$ high heterogeneity). All the ES values were analyzed using fixed-effect models, which were used for calculating pooled ESs (Hedges' $g$ ) and 95\% confidence intervals (CIs) [37]. Small sample size bias was assessed using Egger's test and visually examined with a funnel plot of ES relative to standard error.

After calculating an overall ES for EF, subgroup analyses were also performed based on the specific EF domains (inhibition, updating/working memory, switching, planning), exercise prescriptions variables (frequency, intensity, type, session time, and length), and sample characteristics (age, sex, physical fitness level, and cognitive status). Subgroup analyses are not presented when the number of ESs within a level was fewer than ten. 


\section{Results}

From an initial search, we identified a total of 26,739 potentially relevant citations, which were further reviewed and led to the retrieval of 247 full-text articles. After evaluating these studies relative to inclusion and exclusion criteria, the meta-analytic review included 38 RCT studies. Of these, the author or authors of six studies with incomplete data were contacted by e-mail. The authors of one study [38] were able to provide additional information, but relevant data were not received for five studies [39-43]. Therefore, 33 articles were included in the quantitative synthesis. The flow of the selection process is summarized in Fig. 1.

\subsection{Characteristics of Included Studies}

A total of 33 studies were included in this review and the characteristics of each study are presented in Table 2 . The sample sizes of the RCTs varied considerably, ranging from 18 to 555 participants. The overall sample size for the review was 7,023 participants including both experimental $(n=3606)$ and control $(n=3417)$ participants.

In terms of the sub-domains of EF, the largest number of ESs were for measures of updating/working memory ( $n=42)$, followed by inhibition ( $n=27)$, switching $(n=34)$, and planning $(n=4)$. Regarding exercise prescription variables, the most ESs derived from exercise training interventions offered with a moderate frequency of 3-4 days/week $(n=56)$, performed at a vigorous intensity $(n=44)$, using aerobic exercise $(n=45)$, for a short $(\leq 45 \mathrm{~min})$ session time ( $n=50)$, and over a medium (4-6 months) length of time $(n=49)$.

The average ages of the participants ranged from 62.0 to 85.9 years, with most of the ESs $(n=84)$ deriving from midold adults (66-75 years). The samples in the studies were mostly both male and female participants $(n=72)$, followed by only female $(n=21)$ and only male $(n=14)$. Relatively

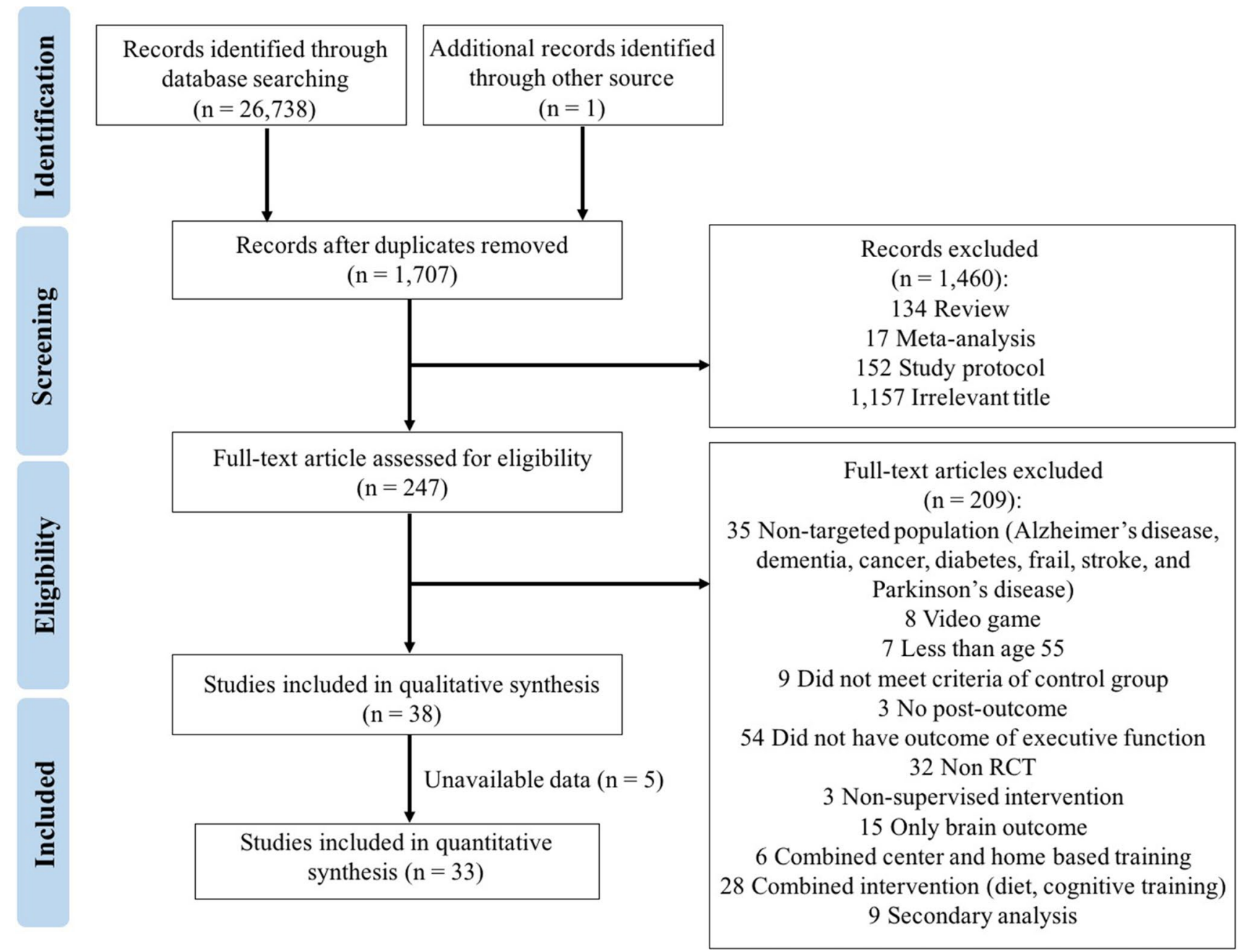

Fig. 1 PRISMA flow chart of the study selection process 
Table 2 Overview characteristics of available evidence from randomized controlled trials on exercise training interventions and executive function

\begin{tabular}{|c|c|c|c|c|}
\hline Study & Grouping & EF Task & Exercise characteristics & Sample characteristics \\
\hline Albinet et al. [64] & $\begin{array}{l}\text { Swimming }(n=21) \\
\text { Stretching }(n=20)\end{array}$ & $\begin{array}{l}\text { Stroop task } \\
\text { RNG } \\
\text { Hayling task } \\
\text { RST } \\
\text { N-back } \\
\text { DST } \\
\text { Plus-Minus task } \\
\text { DLT }\end{array}$ & $\begin{array}{l}\text { Freq.: } 2 \text { days/week } \\
\text { Int.: } 40-65 \% \text { HRR } \\
\text { Type: AE } \\
\text { Time: } 40 \text { min } \\
\text { Length: } 21 \text { weeks }\end{array}$ & $\begin{array}{l}\text { Age: } 60-75 \\
\text { Sex: Both } \\
\text { PF: NR } \\
\text { CS: Normal }\end{array}$ \\
\hline Albinet et al. [65] & $\begin{array}{l}\text { Aerobic }(n=12) \\
\text { Stretching }(n=12)\end{array}$ & WCST & $\begin{array}{l}\text { Freq.: } 3 \text { days/week } \\
\text { Int.: } 40-60 \% \text { HRR } \\
\text { Type: AE } \\
\text { Time: } 40 \text { min } \\
\text { Length: } 12 \text { weeks }\end{array}$ & $\begin{array}{l}\text { Age: } 65-78 \\
\text { Sex: Both } \\
\text { PF: Sedentary } \\
\text { CS: Normal }\end{array}$ \\
\hline Coetsee and Terblanche [66] & $\begin{array}{l}\text { Resistance }(n=22) \\
\text { Aerobic interval }(n=13) \\
\text { Aerobic continuous }(n=13) \\
\text { Control }(n=19)\end{array}$ & Stroop test & $\begin{array}{l}\text { Freq.: } 3 \text { days/week } \\
\text { Int.: } 75-100 \% 10 \mathrm{RM} \text { (RE) } \\
\text { 90-95\% } \mathrm{HR}_{\max }(\mathrm{AE}-\text { interval) } \\
70-75 \% \mathrm{HR}_{\max }(\mathrm{AE}-\text { continuous }) \\
\text { Type: } \mathrm{RE} \text { and } \mathrm{AE} \\
\text { Time: } \mathrm{NR}(\mathrm{RE}) \\
16 \text { min }(90-95 \% \mathrm{AE}-\text { interval) } \\
47 \text { min }(70-75 \% \mathrm{AE}-\text { continuous) } \\
\text { Length: } 16 \text { weeks }\end{array}$ & $\begin{array}{l}\text { Age: } 55-75 \\
\text { Sex: Both } \\
\text { PF: Fit } \\
\text { CS: Normal }\end{array}$ \\
\hline Doi et al. [67] & $\begin{array}{l}\text { Dance }(n=67) \\
\text { Control }(n=67)\end{array}$ & TMT & $\begin{array}{l}\text { Freq.: } 1 \text { day/week } \\
\text { Int.: NR } \\
\text { Type: Dance } \\
\text { Time: } 60 \text { min } \\
\text { Length: } 40 \text { weeks }\end{array}$ & $\begin{array}{l}\text { Age: }>70 \\
\text { Sex: Both } \\
\text { PF: Fit } \\
\text { CS: MCI }\end{array}$ \\
\hline Erickson et al. [68] & $\begin{array}{l}\text { Aerobic }(n=60) \\
\text { Control }(n=60)\end{array}$ & Spatial memory & $\begin{array}{l}\text { Freq.: } \mathrm{NR} \\
\text { Int.: } 60-75 \% \mathrm{HR}_{\max } \\
\text { Type: } \mathrm{AE} \\
\text { Time: } 10-40 \mathrm{~min} \\
\text { Length: } 12 \text { months }\end{array}$ & $\begin{array}{l}\text { Age: } 65.5-67.6 \\
\text { Sex: Both } \\
\text { PF: Sedentary } \\
\text { CS: Normal }\end{array}$ \\
\hline Ferreira et al. [69] & $\begin{array}{l}\text { Walking }(n=22) \\
\text { Control }(n=22)\end{array}$ & WCST & $\begin{array}{l}\text { Freq.: } 3 \text { days/week } \\
\text { Int.: } 60-80 \text { HRR } \\
\text { Type: AE } \\
\text { Time: } 40-50 \text { min } \\
\text { Length: } 6 \text { months }\end{array}$ & $\begin{array}{l}\text { Age: } 60-79 \\
\text { Sex: Both } \\
\text { PF: Fit } \\
\text { CS: Normal }\end{array}$ \\
\hline Gothe et al. [70] & $\begin{array}{l}\text { Yoga }(n=61) \\
\text { Control }(n=57)\end{array}$ & $\begin{array}{l}\text { RST } \\
\text { N-back } \\
\text { Task switching }\end{array}$ & $\begin{array}{l}\text { Freq.: } 3 \text { days/week } \\
\text { Int.: NR } \\
\text { Type: Yoga } \\
\text { Time: } 60 \text { min } \\
\text { Length: } 8 \text { weeks }\end{array}$ & $\begin{array}{l}\text { Age: } 62.02 \\
\text { Sex: Both } \\
\text { PF: Sedentary } \\
\text { CS: Normal }\end{array}$ \\
\hline Gothe et al. [71] & $\begin{array}{l}\text { Yoga }(n=61) \\
\text { Control }(n=57)\end{array}$ & $\begin{array}{l}\text { Task switching } \\
\text { running memory span } \\
\text { N-back }\end{array}$ & $\begin{array}{l}\text { Freq.: } 3 \text { days/week } \\
\text { Int.: NR } \\
\text { Type: Yoga } \\
\text { Time: } 60 \text { min } \\
\text { Length: } 8 \text { weeks }\end{array}$ & $\begin{array}{l}\text { Age: } 55-79 \\
\text { Sex: Both } \\
\text { PF: Sedentary } \\
\text { CS: Normal }\end{array}$ \\
\hline Gothe et al. [72] & $\begin{array}{l}\text { Yoga }(n=61) \\
\text { Control }(n=57)\end{array}$ & TMT & $\begin{array}{l}\text { Freq.: } 3 \text { days/week } \\
\text { Int.: NR } \\
\text { Type: Yoga } \\
\text { Time: NR } \\
\text { Length: } 8 \text { weeks }\end{array}$ & $\begin{array}{l}\text { Age: } 62 \\
\text { Sex: Both } \\
\text { PF: Sedentary } \\
\text { CS: Normal }\end{array}$ \\
\hline Iuliano et al. [73] & $\begin{array}{l}\text { Resistance }(n=20) \\
\text { Cardiovascular }(n=20) \\
\text { Postural }(n=20) \\
\text { Control }(n=20)\end{array}$ & $\begin{array}{l}\text { RPM } \\
\text { Stroop } \\
\text { TMT }\end{array}$ & $\begin{array}{l}\text { Freq.: } 3 \text { days/week } \\
\text { Int.: } \\
\text { 80-85\% 1RM (RE) } \\
\text { 70-80\% HRR (AE) } \\
\text { Type: RE and AE } \\
\text { Time: } 30 \text { min } \\
\text { Length: } 12 \text { weeks }\end{array}$ & $\begin{array}{l}\text { Age: } 66.96 \\
\text { Sex: Both } \\
\text { PF: Sedentary } \\
\text { CS: Normal }\end{array}$ \\
\hline
\end{tabular}


Table 2 (continued)

\begin{tabular}{|c|c|c|c|c|}
\hline Study & Grouping & EF Task & Exercise characteristics & Sample characteristics \\
\hline Kimura et al. [74] & $\begin{array}{l}\text { Strength }(n=65) \\
\text { Control }(n=85)\end{array}$ & Task switching & $\begin{array}{l}\text { Freq.: NR } \\
\text { Int.: } 60 \% 1 \mathrm{RM} \\
\text { Type: Combined exercise } \\
\text { Time: } 1.5 \mathrm{~h} \\
\text { Length: } 12 \text { weeks }\end{array}$ & $\begin{array}{l}\text { Age: }>65 \\
\text { Sex: Both } \\
\text { PF: Fit } \\
\text { CS: Normal }\end{array}$ \\
\hline Klusmann et al. [75] & $\begin{array}{l}\text { Exercise }(n=91) \\
\text { Control }(n=76)\end{array}$ & $\begin{array}{l}\text { VFT } \\
\text { Stroop Test } \\
\text { TMT }\end{array}$ & $\begin{array}{l}\text { Freq.: NR } \\
\text { Int.: NR } \\
\text { Type: Combined exercise } \\
\text { Time: } 90 \text { min } \\
\text { Length: } 24 \text { weeks }\end{array}$ & $\begin{array}{l}\text { Age: } 73.6 \\
\text { Sex: Both } \\
\text { PF: Fit } \\
\text { CS: Normal }\end{array}$ \\
\hline Lam et al. [76] & $\begin{array}{l}\text { Physical }(n=147) \\
\text { Social }(n=131)\end{array}$ & $\begin{array}{l}\text { LDRT } \\
\text { Category Fluency test } \\
\text { Digit span } \\
\text { TMT }\end{array}$ & $\begin{array}{l}\text { Freq.: } 1 \text { day/week } \\
\text { Int.: NR } \\
\text { Type: Combined exercise } \\
\text { Time: NR } \\
\text { Length: } 48 \text { weeks }\end{array}$ & $\begin{array}{l}\text { Age: }>60 \\
\text { Sex: Both } \\
\text { PF: NR } \\
\text { CS: MCI }\end{array}$ \\
\hline Lazarou et al. [77] & $\begin{array}{l}\text { Intervention }(n=89) \\
\text { Control }(n=65)\end{array}$ & $\begin{array}{l}\text { VFT } \\
\text { ROCFT }\end{array}$ & $\begin{array}{l}\text { Freq.: } 2 \text { days/week } \\
\text { Int.: NR } \\
\text { Type: Dance } \\
\text { Time: } 60 \text { min } \\
\text { Length: } 40 \text { weeks }\end{array}$ & $\begin{array}{l}\text { Age: } 55-75 \\
\text { Sex: NR } \\
\text { PF: Fit } \\
\text { CS: MCI }\end{array}$ \\
\hline Leckie et al. [78] & $\begin{array}{l}\text { Walking }(n=47) \\
\text { Control }(n=45)\end{array}$ & Task switching & $\begin{array}{l}\text { Freq.: } 3 \text { days/week } \\
\text { Int.: } 60-75 \% \text { HRR } \\
\text { Type: AE } \\
\text { Time: } 40 \text { min } \\
\text { Length: } 48 \text { weeks }\end{array}$ & $\begin{array}{l}\text { Age: } 66.82 \\
\text { Sex: Both } \\
\text { PF: Sedentary } \\
\text { CS: Normal }\end{array}$ \\
\hline Liu-Ambrose et al. [79] & $\begin{array}{l}\text { RT-1 }(n=20) \\
\text { RT-2 }(n=15) \\
\text { BAT }(n=17)\end{array}$ & Flanker task & $\begin{array}{l}\text { Freq.: } 2 \text { days/week } \\
\text { Int.: NR } \\
\text { Type: RE } \\
\text { Time: NR } \\
\text { Length: } 48 \text { weeks }\end{array}$ & $\begin{array}{l}\text { Age: } 65-75 \\
\text { Sex: F } \\
\text { PF: Fit } \\
\text { CS: Normal }\end{array}$ \\
\hline Liu-Ambrose et al. [80] & $\begin{array}{l}\text { RT-1 }(n=52) \\
\text { RT-2 }(n=54) \\
\text { BAT }(n=49)\end{array}$ & $\begin{array}{l}\text { Stroop test } \\
\text { TMT } \\
\text { VDS }\end{array}$ & $\begin{array}{l}\text { Freq.: } 1-2 \text { days/week } \\
\text { Int.: 6-8 RM } \\
\text { Type: RE } \\
\text { Time: } 60 \text { min } \\
\text { Length: } 48 \text { weeks }\end{array}$ & $\begin{array}{l}\text { Age: } 65-75 \\
\text { Sex: F } \\
\text { PF: Fit } \\
\text { CS: Normal }\end{array}$ \\
\hline Lu et al. [81] & $\begin{array}{l}\text { Tai Chi }(n=15) \\
\text { Control }(n=16)\end{array}$ & Auditory Stroop test & $\begin{array}{l}\text { Freq.: } 3 \text { days/week } \\
\text { Int.: NR } \\
\text { Type: Tai Chi } \\
\text { Time: } 60 \text { min } \\
\text { Length: } 16 \text { weeks }\end{array}$ & $\begin{array}{l}\text { Age: }>65 \\
\text { Sex: F } \\
\text { PF: Fit } \\
\text { CS: Normal }\end{array}$ \\
\hline Lu et al. [81] & $\begin{array}{l}\text { Dumbbell }(n=22) \\
\text { Control }(n=23)\end{array}$ & $\begin{array}{l}\text { TMT } \\
\text { Digit Span }\end{array}$ & $\begin{array}{l}\text { Freq.: } 3 \text { days/week } \\
\text { Int.: NR } \\
\text { Type: } \mathrm{RE} \\
\text { Time: } 60 \text { min } \\
\text { Length: } 12 \text { weeks }\end{array}$ & $\begin{array}{l}\text { Age: }>65 \\
\text { Sex: Both } \\
\text { PF: Fit } \\
\text { CS: MCI }\end{array}$ \\
\hline Mavros et al. [82] & $\begin{array}{l}\text { PRT }(n=27) \\
\text { Control }(n=27)\end{array}$ & $\begin{array}{l}\text { Category fluency } \\
\text { COWAT }\end{array}$ & $\begin{array}{l}\text { Freq.: } 2-3 \text { days/week } \\
\text { Int.: } 80-92 \% 1 \mathrm{RM} \\
\text { Type: RE } \\
\text { Time: } 60-100 \mathrm{~min} \\
\text { Length: } 24 \text { weeks }\end{array}$ & $\begin{array}{l}\text { Age: }>55 \\
\text { Sex: Both } \\
\text { PF: Fit } \\
\text { CS: MCI }\end{array}$ \\
\hline Nagamatsu et al. [83] & $\begin{array}{l}\text { Aerobic }(n=30) \\
\text { Resistance }(n=28) \\
\text { Control }(n=28)\end{array}$ & SWMT & $\begin{array}{l}\text { Freq.: } 2 \text { days/week } \\
\text { Int.: 6-8 RM (RE) } \\
\text { 70-80\% HRR (AE) } \\
\text { Type: RE and AE } \\
\text { Time: } 60 \text { min } \\
\text { Length: } 24 \text { weeks }\end{array}$ & $\begin{array}{l}\text { Age: } 70-80 \\
\text { Sex: F } \\
\text { PF: Fit } \\
\text { CS: MCI }\end{array}$ \\
\hline
\end{tabular}


Table 2 (continued)

\begin{tabular}{|c|c|c|c|c|}
\hline Study & Grouping & EF Task & Exercise characteristics & Sample characteristics \\
\hline Nguyen and Kruse [84] & $\begin{array}{l}\text { Tai Chi }(n=48) \\
\text { Control }(n=48)\end{array}$ & TMT & $\begin{array}{l}\text { Freq.: } 2 \text { days/week } \\
\text { Int.: NR } \\
\text { Type: Tai Chi } \\
\text { Time: } 60 \text { min } \\
\text { Length: } 24 \text { weeks }\end{array}$ & $\begin{array}{l}\text { Age: } 60-79 \\
\text { Sex: Both } \\
\text { PF: NR } \\
\text { CS: Normal }\end{array}$ \\
\hline Nishiguchi et al. [85] & $\begin{array}{l}\text { Exercise }(n=24) \\
\text { Control }(n=24)\end{array}$ & TMT & $\begin{array}{l}\text { Freq.: NR } \\
\text { Int.: NR } \\
\text { Type: AE } \\
\text { Time: } 90 \text { min } \\
\text { Length: } 12 \text { weeks }\end{array}$ & $\begin{array}{l}\text { Age: }>60 \\
\text { Sex: Both } \\
\text { PF: NR } \\
\text { CS: Normal }\end{array}$ \\
\hline Nocera et al. [86] & $\begin{array}{l}\text { Spin }(n=10) \\
\text { Control }(n=8)\end{array}$ & VFT & $\begin{array}{l}\text { Freq.: } 3 \text { days/week } \\
\text { Int.: } 50-75 \% \text { HRR } \\
\text { Type: AE } \\
\text { Time: } 20-45 \text { min } \\
\text { Length: } 12 \text { weeks }\end{array}$ & $\begin{array}{l}\text { Age: } 65-89 \\
\text { Sex: Both } \\
\text { PF: Sedentary } \\
\text { CS: Normal }\end{array}$ \\
\hline Nouchi et al. [87] & $\begin{array}{l}\text { Combination }(n=32) \\
\text { Control }(n=32)\end{array}$ & $\begin{array}{l}\text { VFT } \\
\text { Stroop test } \\
\text { Digit Span }\end{array}$ & $\begin{array}{l}\text { Freq.: } 3 \text { days/week } \\
\text { Int.: } 60-80 \% \mathrm{HR}_{\max } \\
\text { Type: Combined exercise } \\
\text { Time: } 30 \text { min } \\
\text { Length: } 4 \text { weeks }\end{array}$ & $\begin{array}{l}\text { Age: }>60 \\
\text { Sex: Both } \\
\text { PF: Sedentary } \\
\text { CS: Normal }\end{array}$ \\
\hline Oken et al. [51] & $\begin{array}{l}\text { Exercise }(n=47) \\
\text { Yoga }(n=44) \\
\text { Wait list }(n=44)\end{array}$ & Stroop test & $\begin{array}{l}\text { Freq.: } 1 \text { day/week and } 5 \text { days/week } \\
\text { Int.: } 70 \% \mathrm{HR}_{\max } \\
\text { NR } \\
\text { Type: } \\
\text { AE } \\
\text { Yoga } \\
\text { Time: } 60 \text { min (AE) } \\
90 \text { min (Yoga) } \\
\text { Length: } 24 \text { weeks }\end{array}$ & $\begin{array}{l}\text { Age: } 65-85 \\
\text { Sex: Both } \\
\text { PF: Fit } \\
\text { CS: Normal }\end{array}$ \\
\hline Prehn et al. [88] & $\begin{array}{l}\mathrm{AE}(n=11) \\
\mathrm{NAE}(n=18)\end{array}$ & $\begin{array}{l}\text { TMT } \\
\text { Digit span } \\
\text { Stroop test } \\
\text { AVLT }\end{array}$ & $\begin{array}{l}\text { Freq.: } 2 \text { days/week } \\
\text { Int.: } 80 \% \mathrm{AT} \\
\text { Type: AE } \\
\text { Time: } 45 \text { min } \\
\text { Length: } 24 \text { weeks }\end{array}$ & $\begin{array}{l}\text { Age: } 50-80 \\
\text { Sex: Both } \\
\text { PF: Sedentary } \\
\text { CS: Normal }\end{array}$ \\
\hline Smiley-Oyen et al. [89] & $\begin{array}{l}\text { Cardio }(n=52) \\
\text { Flexibility }(n=53)\end{array}$ & $\begin{array}{l}\text { GNG } \\
\text { Stroop test } \\
\text { WCST }\end{array}$ & $\begin{array}{l}\text { Freq.: } 3 \text { days/week } \\
\text { Int.: } 65-80 \% \text { HRR } \\
\text { Type: AE } \\
\text { Time: } 25-30 \text { min } \\
\text { Length: } 40 \text { weeks }\end{array}$ & $\begin{array}{l}\text { Age: } 65-79 \\
\text { Sex: Both } \\
\text { PF: Fit } \\
\text { CS: Normal }\end{array}$ \\
\hline Suzuki et al. [90] & $\begin{array}{l}\text { Exercise }(n=25) \\
\text { Control }(n=25)\end{array}$ & $\begin{array}{l}\text { VFT } \\
\text { Category fluency } \\
\text { Stroop test }\end{array}$ & $\begin{array}{l}\text { Freq.: } 2 \text { days/week } \\
\text { Int.: } 60 \% \text { HRmax } \\
\text { Type: Combined exercise } \\
\text { Time: } 90 \text { min } \\
\text { Length: } 48 \text { weeks }\end{array}$ & $\begin{array}{l}\text { Age: } 65-93 \\
\text { Sex: Both } \\
\text { PF: Sedentary } \\
\text { CS: MCI }\end{array}$ \\
\hline Sungkarat et al. [91] & $\begin{array}{l}\text { Tai Chi }(n=33) \\
\text { Control }(n=33)\end{array}$ & $\begin{array}{l}\text { TMT } \\
\text { Digit span }\end{array}$ & $\begin{array}{l}\text { Freq.: } 3 \text { days/week } \\
\text { Int.: NR } \\
\text { Type: Tai Chi } \\
\text { Time: } 50 \text { min } \\
\text { Length: } 24 \text { weeks }\end{array}$ & $\begin{array}{l}\text { Age: } 68.3 \text { (Tai Chi), } \\
67.5 \text { (Control) } \\
\text { Sex: Both } \\
\text { PF: Sedentary } \\
\text { CS: MCI }\end{array}$ \\
\hline Tsai et al. [38] & $\begin{array}{l}\text { Closed-skill }(n=23) \\
\text { Open-skill }(n=23) \\
\text { Control }(n=23)\end{array}$ & $\begin{array}{l}\text { N-back } \\
\text { Task switching }\end{array}$ & $\begin{array}{l}\text { Freq.: } 3 \text { days/week } \\
\text { Int.: } 70-75 \% \text { (AE) } \\
\text { NR (CE) } \\
\text { Type: AE and CE } \\
\text { Time: } 30 \text { min (AE) } \\
40 \text { min (CE) } \\
\text { Length: } 24 \text { weeks }\end{array}$ & $\begin{array}{l}\text { Age: } 60-80 \\
\text { Sex: M } \\
\text { PF: Sedentary } \\
\text { CS: Normal }\end{array}$ \\
\hline
\end{tabular}


Table 2 (continued)

\begin{tabular}{lllll}
\hline Study & Grouping & EF Task & Exercise characteristics & Sample characteristics \\
\hline Vaughan et al. [92] & Intervention $(n=25)$ & TMT & Freq.: 2 days/week & Age: 65-75 \\
& Control $(n=23)$ & LNS & Int.: NR & Sex: F \\
& & Stroop Test & Type: Combined exercise & PF: Fit \\
& & COWAT & Time: 60 min & CS: Normal \\
van Uffelen et al. [93] & & Length: 16 weeks & Age: 70-80 \\
& Walking $(n=77)$ & VFT & Freq.: 2 days/week & Sex: M and F \\
& Placebo activity $(n=75)$ & & Int:: 3 MET & PF: Fit \\
& & & Type: AE & CS: MCI \\
\hline
\end{tabular}

$N R$ not reported, Freq. frequency, Int. intensity, $P F$ physical fitness level, $C S$ cognitive status, $M$ male, $F$ female, $M C I$ mild cognitive impairment, $A E$ aerobic exercise, $R E$ resistance exercise, $C E$ coordination exercise, $R N G$ the random number generation, GNG go and no go, $W C S T$ Wisconsin card sorting test, $T M T$ trail making test, $R S T$ running span task, $V F T$ verbal fluency test, $R P M$ Raven's progressive matrices Test, $D S T$ the dimension-switching task, $D L T$ the digit-letter task, SWMT Spatial Working Memory task, VDS verbal digit span, $L N S$ the letter-number sequencing, COWAT control oral word association test, ROCFT Rey osterrieth Complex Figure Test, $L D R T$ the list learning delayed recall test, $A V L T$ auditory verbal learning test, $A T$ anaerobic threshold, $M E T$ metabolic equivalents

equal numbers of ESs were calculated when participants were classified as sedentary $(n=46)$ as when they were categorized as physically fit $(n=48)$. Lastly, most ESs derived from individuals with normal cognitive status $(n=85)$, with a smaller number of ESs for participants with MCI $(n=22)$.

The summary of the quality assessment data is presented in Fig. 2. The results showed that quality with respect to three of the criteria (e.g. allocation concealment, incomplete outcome data, selective outcome reporting) was low with over $50 \%$ of the studies having high or unclear risk.

\subsection{Overall Analysis, Heterogeneity and Small Sample Size Bias}

The overall ES revealed by the meta-analysis was small but significant and positive $(g=0.21,95 \%$ CI $0.17,0.26$, $p<0.05)$ with moderate heterogeneity $\left(I^{2}=59.25\right)$ (Table 3$)$.
The funnel plot is shown in Fig. 3. Egger's test and a visual interpretation of the funnel plot suggests that there is no relationship between standard error and ES, suggesting that small sample size has not systematically affected the findings [37].

\subsection{Subgroup Analysis}

\subsubsection{EF Sub-Domains}

The results of the subgroup analyses are shown in Table 3. Four EF sub-domains were included in the meta-analytic review; however, the number of ESs for the sub-domain of planning was fewer than 10 , which did not match the criteria for further analysis. For other EF sub-domains, the results indicated no significant differences among inhibition, updating/working memory, and shifting $[Q(2)=4.33, p>0.05]$.
Fig. 2 Summary of risk of bias for each item presented as a percentage across all included studies
Random sequence generation

$$
\begin{aligned}
& \text { Allocation concealment } \\
& \text { Blinding of participants } \\
& \text { Blinding of assessment } \\
& \text { Incomplete outcome data } \\
& \text { Selective reporting }
\end{aligned}
$$

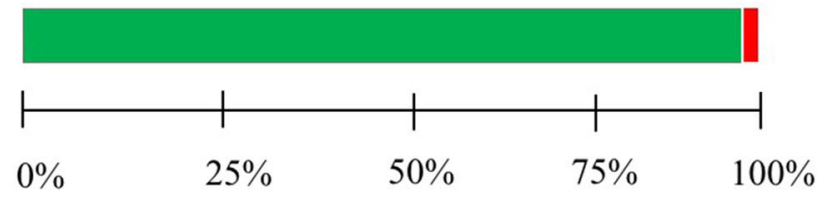

Low risk

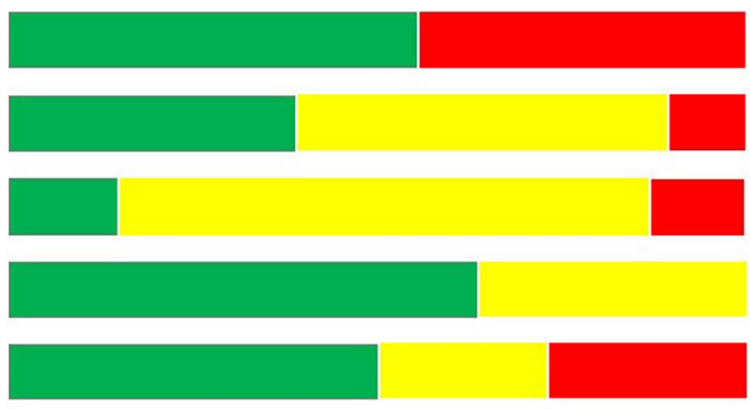

Unclear
High risk 
Table 3 Summary of exercise training intervention effect on executive function (EF). Subgroups denote the variables from EF domain, exercise prescription variables and sample characteristics

\begin{tabular}{|c|c|c|c|c|c|}
\hline \multirow[t]{3}{*}{ Overall } & $Q(d f)$ & \multirow{2}{*}{$\frac{n \mathrm{ES}}{107}$} & \multicolumn{2}{|c|}{ Hedges' $g(95 \% \mathrm{CI})$} & \multirow{2}{*}{$\frac{I^{2}}{59.25}$} \\
\hline & $Q(106)=260.09, p<0.01$ & & 0.2 & 0.02 & \\
\hline & $Q(d f)$ & & $n \mathrm{ES}$ & Hedges' $g(95 \%$ CI $)$ & SE \\
\hline EF dom & $Q(2)$ & & & & \\
\hline Inhibit & & & 27 & $0.14 *(0.04,0.24)$ & 0.05 \\
\hline Updati & & & 42 & $0.19 *(0.12,0.26)$ & 0.04 \\
\hline Shiftin & & & 34 & $0.27 *(0.19,0.36)$ & 0.04 \\
\hline Planni & & & 4 & - & - \\
\hline \multicolumn{6}{|c|}{ Exercise prescription variables } \\
\hline Freque & $Q(1)$ & & & & \\
\hline Low & & & 46 & $0.15 *(0.08,0.22)$ & 0.04 \\
\hline Mod & nes) & & 56 & $0.31 *(0.24,0.38)$ & 0.04 \\
\hline Intensi & $Q(1)$ & & & & \\
\hline Mod & & & 25 & $0.11 *(0.00,0.21)$ & 0.05 \\
\hline Vigo & & & 44 & $0.22 *(0.14,0.30)$ & 0.04 \\
\hline Type & $Q(4)$ & & & & \\
\hline Aero & & & 45 & $0.14 *(0.06,0.23)$ & 0.04 \\
\hline Resi & & & 20 & $0.22 *(0.10,0.33)$ & 0.06 \\
\hline Tai $C$ & & & 14 & $0.38 *(0.27,0.49)$ & 0.06 \\
\hline Com & & & 18 & $0.10 *(0.00,0.19)$ & 0.05 \\
\hline Othe & ercise & & 10 & $0.44 *(0.29,0.60)$ & 0.05 \\
\hline Sessiol & $Q(2)$ & & & & \\
\hline Shor & & & 50 & $0.26 *(0.18,0.33)$ & 0.04 \\
\hline Mod & $\min )$ & & 39 & $0.26 *(0.18,0.33)$ & 0.07 \\
\hline Long & & & 10 & $0.30 *(0.15,0.44)$ & 0.07 \\
\hline Length & $Q(2)$ & & & & \\
\hline Shor & & & 29 & $0.32 *(0.23,0.41)$ & 0.05 \\
\hline Medi & ath) & & 49 & $0.26 *(0.18,0.34)$ & 0.04 \\
\hline Long & & & 29 & $0.09 *(0.01,0.17)$ & 0.04 \\
\hline \multicolumn{6}{|c|}{ Sample characteristics } \\
\hline Age & $Q(2)$ & & & & \\
\hline Youn & yrs) & & 11 & $0.30 *(0.18,0.43)$ & 0.06 \\
\hline Mid- & & & 84 & $0.25^{*}(0.19,0.31)$ & 0.03 \\
\hline Old-c & & & 10 & $-0.05(-0.17,0.08)$ & 0.06 \\
\hline Sex & $Q(2)$ & & & & \\
\hline Only & & & 14 & $0.33 *(0.18,0.48)$ & 0.08 \\
\hline Only & & & 21 & $0.29 *(0.17,0.40)$ & 0.06 \\
\hline Both & & & 72 & $0.18 *(0.12,0.23)$ & 0.03 \\
\hline Physic & $Q(1)$ & & & & \\
\hline Sede & & & 46 & $0.33 *(0.25,0.41)$ & 0.04 \\
\hline Fit & & & 48 & $0.16 *(0.09,0.23)$ & 0.03 \\
\hline Cognit & $Q(1)$ & & & & \\
\hline Norn & & & 85 & $0.26^{*}(0.20,0.32)$ & 0.03 \\
\hline MCI & & & 22 & $0.08 *(0.00,0.17)$ & 0.05 \\
\hline
\end{tabular}

$n E S$ numbers of effect size, $S E$ standard error, $I^{2}$ I square, yrs years, $E F$ executive function, $W M$ working memory, $M C I$ mild cognitive impairment

*Represents $p<0.05$ when comparing the effect size to zero 
Fig. 3 Funnel plot for visual inspection of study bias

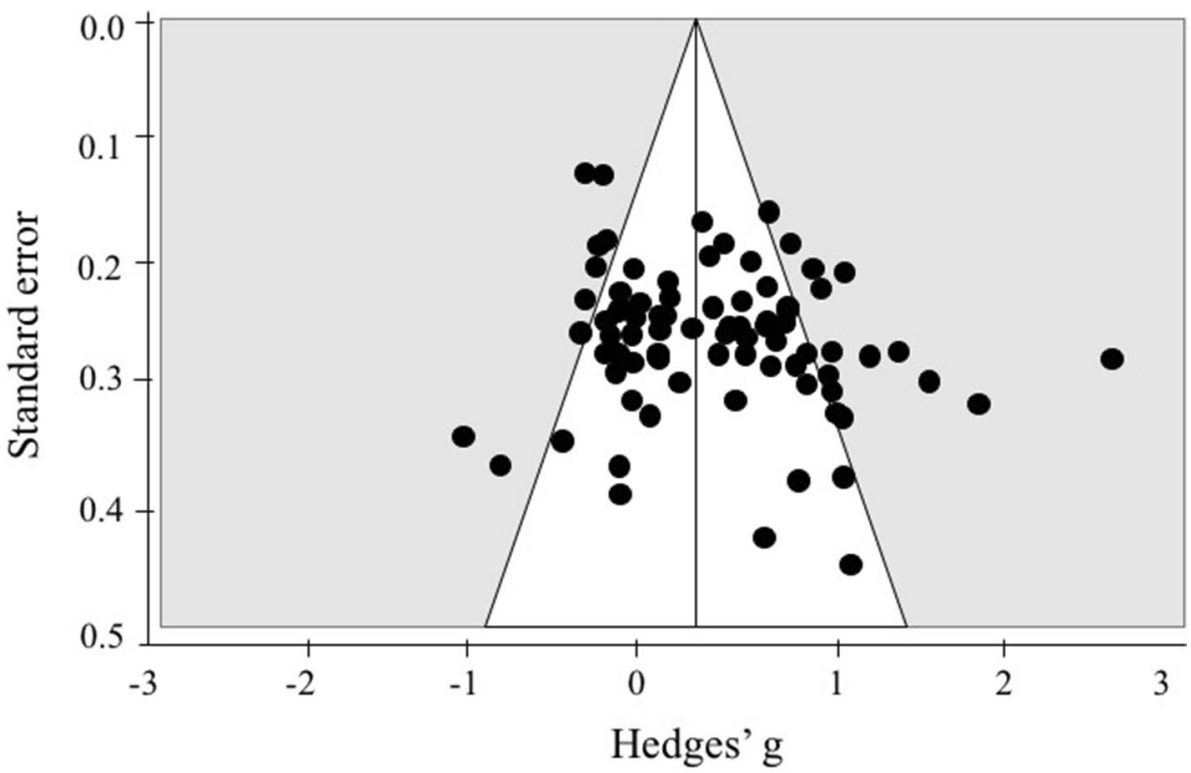

\subsubsection{Exercise Prescription Variables}

The frequency of exercise training interventions significantly moderated the effect of exercise on $\mathrm{EF}[Q(1)=10.86$, $p<0.05]$. The results of the subgroup analysis indicated that the ES for older adults engaged in exercise training interventions of moderate frequency (3-4 times per week) ( $g=0.31$, $p<0.05)$ was larger than that for low frequency (1-2 times per week) $(g=0.15, p<0.05)$. The subgroup analysis indicated that type of exercise training $[Q(4)=26.18, p<0.05]$ significantly moderated the effect of exercise on EF, showing ESs for older adults engaged in exercise training interventions of other forms $(g=0.44, p<0.05)$ were largest, followed by Tai Chi and yoga $(g=0.38, p<0.05)$, resistance exercise $(g=0.22, p<0.05)$, aerobic exercise $(g=0.14$, $p<0.05)$, and combined exercise $(g=0.10, p<0.05)$. In addition, the results of subgroup analysis also showed that exercise length $[Q(2)=16.64, p<0.05]$ significantly moderated the effect of exercise on EF, revealing ESs were largest for short length (1-3 month) exercise training $(g=0.32)$, followed by mid length (4-6 month) exercise training ( $g=0.26)$, and long length ( $>6$ month) exercise training $(\mathrm{g}=0.09)$.

There were no significant differences among the ESs based upon intensity level $[Q(1)=2.87, p>0.05]$ and exercise session time $[Q(2)=0.21, p>0.05]$.

\subsubsection{Sample Characteristics}

The subgroup analysis indicated age significantly moderated the effects of exercise on $\operatorname{EF}[Q(2)=20.64, p<0.05]$, such that ESs for young-old participants (55-65 years old) $(g=0.30, p<0.05)$ and mid-old participants $(66-75$ years old) $(g=0.25, p<0.05)$ were significant. In contrast, studies with old-old participants (more than 76 years old) exhibited negligible ESs $(g=-0.05, p>0.05)$. Additionally, the subgroup analysis indicated significant differences in the ESs as a function of physical fitness levels $[Q(1)=10.80$, $p<0.05]$ and indicated that sedentary participants $(g=0.33$, $p<0.05)$ had a greater relative improvement in EF than fit participants $(g=0.16, p<0.05)$. Furthermore, the subgroup analysis also showed a significant difference in the ESs as a function of cognitive status $[Q(1)=11.44, p<0.05]$, suggesting that ESs were larger for participants with cognitively normal $(g=0.26, p<0.05)$ as compared to those with MCI $(g=0.08, p<0.05)$.

The results of the subgroup analysis showed no significant differences as a function of $\operatorname{sex}[Q(2)=5.38, p>0.05]$.

\section{Discussion}

To our knowledge, this is the first meta-analysis of RCTs to have investigated the effects of exercise training interventions specifically on EF and its sub-domains among older adults. This is important because of the evidence that the largest benefits of exercise training interventions for older adults may be apparent for EF [12]. In addition, no previous meta-analytic reviews have examined if exercise training influences specific domains of EF [13, 14], and it is critical to advance our understanding of moderators important for predicting the magnitude of these benefits.

In this meta-analysis, we analyzed the results of 33 RCT studies and demonstrated that exercise training interventions improve EF with a positive, significant, small ES. According to the overall results of the current meta-analytic review, 
we suggest that exercise training interventions are an effective approach to improving EF in older populations, a view which is generally consistent with the results of several previous meta-analyses [12, 14, 44]. Although the results of this review were similar to those of previous meta-analyses in terms of the pattern of positive effects of exercise training interventions on $\mathrm{EF}$, it is important to recognize that the overall effect obtained was considered small in magnitude. This is a contrast to the moderate ES reported in Colcombe and Kramer [12] and is more consistent with the small ES shown in two other meta-analyses [14, 44].

\subsection{EF Sub-Domains}

Despite previous studies showing the positive effects of exercise training interventions on EF in older adults [12, $14,44]$, this meta-analysis provides an important extension to the literature by exploring the effects of exercise training interventions on sub-domains of EF. The results of the subgroup analysis indicated that older adults engaged in exercise training interventions exhibited small to moderate significant improvements within three specific domains of EF. These domains were inhibition, updating/working memory, and switching. Consistent with a recent meta-analytic review that examined the effects of regular exercise training on $\mathrm{EF}$ in children and reported small improvements in inhibition [45], we showed small effects of exercise training interventions on inhibition in older adults. In addition, we also demonstrated that exercise training interventions for older adults resulted in positive effects on other aspects of EF (i.e., updating/working memory, switching). The findings overall suggest that exercise training interventions influenced multiple aspects of EF.

However, we were not able to present a robust average ES for planning due to the small number of ESs available $(n=4)$, which did not match the criteria for inclusion in this meta-analytic review. Despite the small number of previous studies that have focused on planning, we suggest that the planning aspect of EF should be studied in the future due to the domain being equally important as other EFs (e.g. inhibition, updating/working memory, switching) for older populations [46]. Additionally, several cross-sectional and intervention studies have shown a positive relationship between exercise training interventions and planning in children [47-50], suggesting the possibility that exercise training interventions may also benefit from planning in an older population.

\subsection{Exercise Prescription Variable}

The present meta-analytic review constitutes an important step in evaluating the effects of another group of moderators (e.g. exercise prescription) on the effects of exercise training interventions on EF. The findings indicated that program frequency is a moderator that influences EF effects, such that larger benefits are evident with moderate frequency (3-4 times) exercise as compared to low frequency (1-2 times) exercise. Unfortunately, there were an insufficient number of studies $(n=1)$ [51] testing the effects of high frequency exercise (more than 5 times per week) to allow for a statistical examination of the ES for these studies. Although we found differences in ESs as a function of the frequency of the exercise sessions, it is important to acknowledge that our finding of a positive benefit for both low and moderate frequency exercise is consistent with the results of Northey et al. [14] who suggested that exercise performed at low and moderate frequencies benefits global cognition.

Another issue addressed in this review was examining the effect of exercise intensity on the EF outcomes. The results of this meta-analysis showed a small ES of exercise training intervention on EF regardless of intensity levels, suggesting that exercise training interventions of moderate or vigorous intensity yielded positive improvements in terms of EF. Consistent with a previous meta-analysis which demonstrated that moderate and vigorous intensity exercise training interventions might be an effective alternative for improving global cognitive function [14], our findings further expand the understanding of the positive effect of such interventions specifically on EF among older adults.

The current meta-analysis indicated that exercise training interventions yielded positive improvements in EF regardless of the exercise type, thereby confirming the results of a previous meta-analysis of RCTs in older adults [12]. Importantly, however, our results showed that other forms of exercise training (e.g., dance, coordination exercises) exhibited the largest ES, followed by Tai Chi and yoga, resistance exercise, combined exercise, and aerobic exercise. Notably, these results should be interpreted with caution due to the relatively small number of ESs for all exercise types (i.e., Tai Chi and yoga, combined exercise, resistance, and other) other than aerobic exercise.

For aerobic exercise, our results were similar to Angevaren et al. [52] who reported that RCTs of aerobic exercise training were associated with positive improvements in cognition (e.g. attentional processes, cognitive speed, and motor functions). Similarly, aerobic exercise in this review was found to produce small improvements in EF among older populations.

We also observed positive improvements in EF when older adults engaged in resistance exercise training. These results are consistent with a previous meta-analytic review suggesting that resistance exercise yields positive improvements in terms of the cognitive functions of older adults [14]. Furthermore, we included studies testing the effects of Tai Chi and yoga, since we considered these exercises to have the same functions in terms of enhancing motor fitness 
(e.g. balance, flexibility, and agility). Importantly, the cognitive benefits of these exercise programs were significantly larger than were observed for aerobic exercise. These types of exercise might be considered to be unique in that they are sometimes categorized as mind-body exercises and have been shown to influence different aspects of physical fitness (e.g., flexibility, balance) as compared to aerobic exercise [35]. Although a previous meta-analysis revealed no effects of yoga on global cognitive function [14], our meta-analysis provides evidence of higher ES for EF from the prescription of both Tai Chi and yoga in older populations. This is an important finding with regard to exercise training interventions, because Tai Chi and yoga have been described as being more suitable than some other types of exercise for older adults because of the emphasis on slow controlled movements that result in a minimal risk of injury $[53,54]$.

A previous meta-analysis showed that combined exercise programs have moderate effects on global cognitive function [12]; the results of this review indicated that combined exercise programs have a small effect on EF. Combined exercise in the present review was defined as the combination of two or more types of exercise training as an intervention; therefore, it was more broad than the categories used in previous meta-analyses which only combined aerobic exercise and resistance exercise $[12,14]$. Furthermore, we also included additional exercise types (e.g. dance, coordination exercise) in the review (categorized as 'other') and the ESs were the largest observed. Given that it is important to provide exercise guidelines for older populations, including strategies to maintain and improve EF, future RCTs of exercise training interventions focusing on combined exercise trainings are needed to confirm the findings of the present review.

Lastly, this meta-analytic review also examined differences in ES as a function of the time of exercise training interventions. The results of the subgroup analyses significant improvement in $\mathrm{EF}$ as a function of the session time or length of the program across studies with session times that ranged from $\leq 45$ to $>60$ min and program lengths from 1 months to more than 6 months. This suggests that within these ranges, exercise training interventions of any session time and length result in positive effects for EF in older adults, as suggested in previous reviews [12,14]. Although these meta-analytic results suggest that the specifics in terms of the time of exercise training interventions are not critical for demonstrating improvements in EF, it is important for dose-response studies to be conducted to more formally test the effects of exercise time on the EF benefits observed in an older population.

\subsection{Sample Characteristics}

A previous meta-analysis reported that RCTs of aerobic exercise training resulted in moderate to large positive effects on global cognition among all ages of older adults [12]. The results of the present meta-analysis are also positive but show smaller effects on EF. Given that significant differences were observed between age groups, these findings highlight that exercise training interventions are a strategy to benefit EF in specific age groups of older adults. This might then suggest that exercise is particularly beneficial for EF in relatively younger groups of older adults. However, these results should be interpreted with caution because the number of ESs for the mid-old (66-75) participants was considerably larger than the young-old (55-65) and old-old (>76) participants.

Previous studies have shown that some psychological factors that are associated with biological sex (e.g., steroid hormones, brain-derived neurotrophic factor) may impact cognitive performance [55-57] Thus, we compared studies using single sex samples (male or female) with studies including both sexes. Our findings showed positive effects of exercise on EF in male and female participants tested in single-sex study samples and positive improvements in EF in studies combining both male and female participants. These findings were consistent with those of a previous meta-analysis [12], which suggested no different benefits in cognitive function in those studies including single sex (i.e. male and female) or both sexes. However, this is in contrast to a recent meta-analysis which suggested that some types of exercise training (aerobic exercise, resistance exercise, and combined exercises) had positive and large ESs in studies including higher percentages of female participants $[13,58]$. An important feature differentiating this review from previous reviews is that our data focus only on EF and suggest that exercise training interventions can benefit EF in both male and female participants, with the ES being similar for both sexes.

Another issue was to specifically examine the effects of exercise training interventions on EF in older adults with different physical fitness levels. Interestingly, the subgroup analysis suggested that the ES for sedentary participants was significantly greater than that for participants with higher physical fitness levels. Accordingly, this would imply that older adults with a sedentary lifestyle who participate in regular exercise training would experience greater benefits in terms of EF. This finding supports previous reviews which suggested that exercise in those with lower physical fitness levels might be more beneficial to $\mathrm{EF}$ as compared to such activity in those with high physical fitness levels $[59,60]$. However, while the ESs for sedentary and fit participants were significantly different, the findings of this review suggested a positive effect on EF regardless of fitness levels.

Regarding cognitive status, our result is consistent with previous findings, showing significant improvement in EF among cognitively normal older adults and among older adults with MCI [14, 61-63]. Although the ES of older 
adults with normal cognition is significant higher than those with MCI, the implication of this finding is that exercise can be beneficial even for older adults who have begun to show evidence of clinical cognitive impairment. Taken together, previous and present meta-analyses suggest that exercise training interventions have the capacity to improve EF in older adults with MCI.

\subsection{Strengths and Limitations}

In this meta-analytic review, the primary strength was the inclusion of RCT studies. Because the inclusion criteria required an experimental design, the results may be interpreted in terms of causal relationships. Another strength is the consideration of various key moderators such as the specific EF sub-domain, exercise prescription variables, and sample characteristics. This provides a much clearer picture of the complexity of the relationship between exercise training interventions and $\mathrm{EF}$ than presented in previous reviews.

The present meta-analysis had four limitations, which should be considered in future research. First, because our goal was to update our knowledge of the effects of exercise on cognitive performance looking specifically at subdomains of EF, we limited our search to publications since 2003 when Colcombe and Kramer first identified an overall positive ES for studies focused on older adults and EF [12]. Second, our data indicated that the quality of the studies reviewed was generally poor. Although all of the studies were RCTs, in general the studies did not control for potential risks of bias or failed to report the extent to which they did control for these risks and, hence, are open to alternative interpretations of the findings. Third, some moderators (i.e., EF sub-domain: planning; exercise frequency: high) were excluded from the analysis because fewer than $10 \mathrm{ESs}$ were available overall and there were some moderator levels that were exactly at this minimum number of ESs (e.g., length of exercise session $>60 \mathrm{~min}$ ). Although a meta-analysis does not require a specific number of ESs, a small number of ESs may limit the precision of pooled estimates and the power to detect effects, thus necessitating a priori decisions of a minimum number of ES for inclusion in analyses. Setting a limit of $10 \mathrm{ESs}$ provides some control over this concern, but findings for moderator levels with smaller number of ESs must be considered to be somewhat tentative. Finally, previous reviews examining the outcome of both cognitive and physical function have suggested that the focus of a study in terms of its primary and secondary outcomes may moderate the results of the effects of exercise on cognition [1]. However, most of the studies included in this meta-analytic review examined EF as a primary outcome, making it impossible to examine primary versus secondary outcome as a moderator. In addition, because studies are often powered to detect only the primary outcomes, it may be valuable in the future to consider this as a potential moderator.

\section{Conclusion}

The results of this review demonstrated that exercise training interventions are a promising way to promote overall $\mathrm{EF}$, including inhibition, updating/working memory, and shifting, with small positive effects. Additionally, we observed that exercise training interventions of low and moderate frequency yielded improvements in EF, while also finding positive effects for all types of exercise training and positive effects for interventions at any intensity (i.e., moderate, vigorous) and length (i.e., 1 months to more than 6 months). Regardless of the exercise session time, the size of the effects of exercise training interventions on EF were similar. Last, the results suggested that, regardless of health status, older individuals who were initially sedentary appeared to benefit from exercise training interventions in terms of EF.

Acknowledgements The authors thank Chien-Heng Chu, Chun-Chih Wang, Chih-Han Wu, and Jui-Ti Nien for their contributions to data preparation.

Author Contributions F-TC, JE and Y-KC were responsible for the conception, design and hypothesis of the research. F-TC, JE and Y-KC completed all statistical analyses and all authors wrote the initial drafts of the manuscript. F-TC and Y-KC conducted the systematic search, data extraction, and the assessment of risk of bias. All authors contributed to the drafting of the manuscript and its revisions, including the final version, in relation to intellectual content.

Data Availability Statement All data generated or analyzed during this review are included in this published article (and its supplementary information files).

\section{Compliance with Ethical Standards}

Funding This research was funded by a portion of grant from the Ministry of Science and Technology in Taiwan (MOST 105-2628-H-179001-MY3, 107-2628-H-003-003-MY3, 107-2627-H-003-001-MT5) and the National Taiwan Normal University from the Higher Education Sprout Project by the Ministry of Education in Taiwan.

Conflict of interest Feng-Tzu Chen, Jennifer Etnier, Kuei-Hui Chan, Ping-Kun Chiu, Tsung-Min Hung and Yu-Kai Chang have no conflicts of interest relevant to the content of this review.

Open Access This article is licensed under a Creative Commons Attribution 4.0 International License, which permits use, sharing, adaptation, distribution and reproduction in any medium or format, as long as you give appropriate credit to the original author(s) and the source, provide a link to the Creative Commons licence, and indicate if changes were made. The images or other third party material in this article are included in the article's Creative Commons licence, unless indicated otherwise in a credit line to the material. If material is not included in the article's Creative Commons licence and your intended use is not permitted by statutory regulation or exceeds the permitted use, you will 
need to obtain permission directly from the copyright holder. To view a copy of this licence, visit http://creativecommons.org/licenses/by/4.0/.

\section{References}

1. Falck RS, Landry GJ, Best JR, Davis JC, Chiu BK, Liu-Ambrose T. Cross-sectional relationships of physical activity and sedentary behavior with cognitive function in older adults with probable mild cognitive impairment. Phys Ther. 2017;97(10):975-84.

2. Engeroff T, Ingmann T, Banzer W. Physical activity throughout the adult life span and domain-specific cognitive function in old age: a systematic review of cross-sectional and longitudinal data. Sports Med. 2018;48(6):1405-36.

3. Bento-Torres J, Bento-Torres NVO, Stillman CM, Grove GA Jr, Huang H, Uyar F, et al. Associations between cardiorespiratory fitness, physical activity, intraindividual variability in behavior, and cingulate cortex in younger adults. J Sport Health Sci. 2019;8(4):315-24.

4. Pindus DM, Drollette ES, Raine LB, Kao SC, Khan N, Westfall $\mathrm{DR}$, et al. Moving fast, thinking fast: the relations of physical activity levels and bouts to neuroelectric indices of inhibitory control in preadolescents. J Sport Health Sci. 2019;8(4):301-14.

5. Brini S, Sohrabi HR, Peiffer JJ, Karrasch M, Hamalainen H, Martins RN, et al. Physical activity in preventing alzheimer's disease and cognitive decline: a narrative review. Sports Med. 2018;48(1):29-44.

6. Etnier JL. Physical activity in the prevention of Alzheimer's disease. Kinesiol Rev. 2015;4:28-38.

7. Chen FT, Chan KH, Feng SH, Wu TW, Kao SC, Chang YK. New perspective: effect of exercise and nutritional supplements on cognitive function in older adults. J Phys Educ. 2019;52(S):1-15.

8. Etnier JL, Chang YK. Exercise, cognitive function, and the brain: advancing our understanding of complex relationships. J Sport Health Sci. 2019;8(4):299-300.

9. Audiffren M, Andre N. The exercise-cognition relationship: a virtuous circle. J Sport Health Sci. 2019;8(4):339-47.

10. Blondell SJ, Hammersley-Mather R, Veerman JL. Does physical activity prevent cognitive decline and dementia?: a systematic review and meta-analysis of longitudinal studies. BMC Public Health. 2014;14:510.

11. Kelly ME, Loughrey D, Lawlor BA, Robertson IH, Walsh C, Brennan S. The impact of exercise on the cognitive functioning of healthy older adults: a systematic review and meta-analysis. Ageing Res Rev. 2014;16:12-311.

12. Colcombe S, Kramer AF. Fitness effects on the cognitive function of older adults: a meta-analytic study. Psychol Sci. 2003;14(2):125-30.

13. Falck RS, Davis JC, Best JR, Crockett RA, Liu-Ambrose T. Impact of exercise training on physical and cognitive function among older adults: a systematic review and meta-analysis. Neurobiol Aging. 2019;79:119-30.

14. Northey JM, Cherbuin N, Pumpa KL, Smee DJ, Rattray B. Exercise interventions for cognitive function in adults older than 50: a systematic review with meta-analysis. Br J Sports Med. 2017;52(3):154-60.

15. Banich MT. Executive function: the search for an integrated account. Curr Dir Psychol Sci. 2009;18(2):89-94.

16. Bishop NA, Lu T, Yankner BA. Neural mechanisms of ageing and cognitive decline. Nature. 2010;464(7288):529-35.

17. Rosen ML, Amso D, McLaughlin KA. The role of the visual association cortex in scaffolding prefrontal cortex development: a novel mechanism linking socioeconomic status and executive function. Dev Cogn Neurosci. 2019;39:100699.
18. Shaked D, Katzel LI, Seliger SL, Gullapalli RP, Davatzikos C, Erus G, et al. Dorsolateral prefrontal cortex volume as a mediator between socioeconomic status and executive function. Neuropsychology. 2018;32(8):985-95.

19. Haeger A, Costa AS, Schulz JB, Reetz K. Cerebral changes improved by physical activity during cognitive decline: a systematic review on MRI studies. Neuroimage Clin. 2019;23:101933.

20. Chen FT, Chen YP, Schneider S, Kao SC, Huang CM, Chang YK. Effects of exercise modes on neural processing of working memory in late middle-aged adults: an fMRI study. Front Aging Neurosci. 2019;11:224.

21. Best JR, Chiu BK, Hall PA, Liu-Ambrose T. Larger lateral prefrontal cortex volume predicts better exercise adherence among older women: evidence from two exercise training studies. J Gerontol A Biol Sci Med Sci. 2017;72(6):804-10.

22. Ehlers DK, Daugherty AM, Burzynska AZ, Fanning J, Awick EA, Chaddock-Heyman L, et al. Regional brain volumes moderate, but do not mediate, the effects of group-based exercise training on reductions in loneliness in older adults. Front Aging Neurosci. 2017;9:110.

23. Miyake A, Friedman NP, Emerson MJ, Witzki AH, Howerter A, Wager TD. The unity and diversity of executive functions and their contributions to complex "Frontal Lobe" tasks: a latent variable analysis. Cogn Psychol. 2000;41(1):49-100.

24. Romine CB, Reynolds CR. A model of the development of frontal lobe functioning: findings from a meta-analysis. Appl Neuropsychol. 2005;12(4):190-201.

25. Diamond A, Ling DS. Conclusions about interventions, programs, and approaches for improving executive functions that appear justified and those that, despite much hype, do not. Dev Cogn Neurosci. 2016;18:34-48.

26. Hillman CH, McAuley E, Erickson KI, Liu-Ambrose T, Kramer AF. On mindful and mindless physical activity and executive function: a response to Diamond and Ling (2016). Dev Cogn Neurosci. 2019;37:100529.

27. American College of Sports Medicine. ACSM's guidelines for exercise testing and prescription. 10th ed. New York: Lippincott Williams and Wilkins; 2018.

28. Kramer AF, Erickson KI. Capitalizing on cortical plasticity: influence of physical activity on cognition and brain function. Trends Cogn Sci. 2007;11(8):342-8.

29. Tomporowski PD, Davis CL, Miller PH, Naglieri JA. Exercise and children's intelligence, cognition, and academic cchievement. Educ Psychol Rev. 2008;20(2):111-31.

30. Chang YK, Pan CY, Chen FT, Tsai CL, Huang CC. Effect of resistance-exercise training on cognitive function in healthy older adults: a review. J Aging Phys Act. 2012;20(4):497-517.

31. Liberati A, Altman DG, Tetzlaff J, Mulrow C, Gotzsche PC, Ioannidis JP, et al. The PRISMA statement for reporting systematic reviews and meta-analyses of studies that evaluate healthcare interventions: explanation and elaboration. BMJ. 2009;339:b2700.

32. Higgins JPT, Green S. Cochrane handbook for systematic reviews of interventions. New York: Wiley; 2011.

33. Norton K, Norton L, Sadgrove D. Position statement on physical activity and exercise intensity terminology. J Sports Sci Med. 2010;13(5):496-502.

34. Oberlin LE, Waiwood AM, Cumming TB, Marsland AL, Bernhardt J, Erickson KI. Effects of physical activity on poststroke cognitive function: a meta-analysis of randomized controlled trials. Stroke. 2017;48(11):3093-100.

35. Chan JSY, Deng K, Wu J, Yan JH. Effects of meditation and mindbody exercises on older adults' cognitive performance: a metaanalysis. Gerontologist. 2019;59(6):e782-e790790.

36. Cohen J. Statistical power analysis for the behavioural sciences. 2nd ed. Hillsdale: Lawrence Erlbaum Associates; 1988. 
37. Egger M, Davey Smith G, Schneider M, Minder C. Bias in meta-analysis detected by a simple, graphical test. BMJ. 1997;315(7109):629-34.

38. Tsai CL, Pan CY, Chen FC, Tseng YT. Open- and closed-skill exercise interventions produce different neurocognitive effects on executive functions in the elderly: a 6-month randomized, controlled trial. Front Aging Neurosci. 2017;9:294.

39. Baniqued PL, Gallen CL, Voss MW, Burzynska AZ, Wong CN, Cooke GE, et al. Brain network modularity predicts exerciserelated executive function gains in older adults. Front Aging Neurosci. 2018;9:426.

40. Evers A, Klusmann V, Schwarzer R, Heuser I. Improving cognition by adherence to physical or mental exercise: a moderated mediation analysis. Aging Ment Health. 2011;15(4):446-55.

41. Bolandzadeh N, Tam R, Handy TC, Nagamatsu LS, Hsu CL, Davis JC, et al. Resistance training and white matter lesion progression in older women: exploratory analysis of a 12-month randomized controlled trial. J Am Geriatr Soc. 2015;63(10):2052-60.

42. Baker LD, Frank LL, Foster-Schubert K, Green PS, Wilkinson $\mathrm{CW}$, McTiernan A, et al. Effects of aerobic exercise on mild cognitive impairment: a controlled trial. Arch Neurol. 2010;67(1):71-9.

43. Colcombe SJ, Kramer AF, Erickson KI, Scalf P, McAuley E, Cohen NJ, et al. Cardiovascular fitness, cortical plasticity, and aging. Proc Natl Acad Sci U S A. 2004;101(9):3316-21.

44. Smith PJ, Blumenthal JA, Hoffman BM, Cooper H, Strauman TA, Welsh-Bohmer K, et al. Aerobic exercise and neurocognitive performance: a meta-analytic review of randomized controlled trials. Psychosom Med. 2010;72(3):239-52.

45. Xue Y, Yang Y, Huang T. Effects of chronic exercise interventions on executive function among children and adolescents: a systematic review with meta-analysis. Br J Sports Med. 2019;53:1397-404.

46. Weakley A, Schmitter-Edgecombe M. Naturalistic assessment of task interruption in individuals with mild cognitive impairment. Neuropsychol. 2019;33(1):1-12.

47. van der Niet AG, Smith J, Scherder EJ, Oosterlaan J, Hartman E, Visscher C. Associations between daily physical activity and executive functioning in primary school-aged children. J Sci Med Sport. 2015;18(6):673-7.

48. Gapin J, Etnier JL. The relationship between physical activity and executive function performance in children with attention-deficit hyperactivity disorder. J Sport Exerc Psychol. 2010;32(6):753-63.

49. Zimmer P, Binnebossel S, Bloch W, Hubner ST, Schenk A, Predel HG, et al. Exhaustive exercise alters thinking times in a Tower of London task in a time-dependent manner. Front Physiol. 2016;7:694.

50. Chen FT, Chen SR, Chu IH, Liu JH, Chang YK. Multicomponent exercise intervention and metacognition in obese preadolescents: a randomized controlled study. J Sport Exerc Psychol. 2017;39(4):302-12.

51. Oken BS, Zajdel D, Kishiyama S, Flegal K, Dehen C, Haas M, et al. Randomized, controlled, six-month trial of yoga in healthy seniors: effects on cognition and quality of life. Altern Ther Health Med. 2006;12(1):40-7.

52. Angevaren M, Aufdemkampe G, Verhaar HJ, Aleman A, Vanhees L. Physical activity and enhanced fitness to improve cognitive function in older people without known cognitive impairment. Cochrane Database Syst Rev. 2008(3):Cd005381.

53. Lomas-Vega R, Obrero-Gaitan E, Molina-Ortega FJ, Del-PinoCasado R. Tai Chi for risk of falls. a meta-analysis. J Am Geriatr Soc. 2017;65(9):2037-43.

54. Green E, Huynh A, Broussard L, Zunker B, Matthews J, Hilton CL, et al. Systematic review of yoga and balance: effect on adults with neuromuscular impairment. Am J Occup Ther. 2019;73(1):7301205150p1-7301205150p11.
55. Okamoto M, Hojo Y, Inoue K, Matsui T, Kawato S, McEwen BS, et al. Mild exercise increases dihydrotestosterone in hippocampus providing evidence for androgenic mediation of neurogenesis. Proc Natl Acad Sci USA. 2012;109(32):13100-5.

56. Bakos J, Hlavacova N, Rajman M, Ondicova K, Koros C, Kitraki E, et al. Enriched environment influences hormonal status and hippocampal brain derived neurotrophic factor in a sex dependent manner. Neuroscience. 2009;164(2):788-97.

57. Zhu SW, Yee BK, Nyffeler M, Winblad B, Feldon J, Mohammed $\mathrm{AH}$. Influence of differential housing on emotional behaviour and neurotrophin levels in mice. Behav Brain Res. 2006;169(1):10-20.

58. Barha CK, Falck RS, Davis JC, Nagamatsu LS, Liu-Ambrose T. Sex differences in aerobic exercise efficacy to improve cognition: a systematic review and meta-analysis of studies in older rodents. Front Neuroendocrinol. 2017;46:86-105.

59. Drollette ES, Scudder MR, Raine LB, Moore RD, Saliba BJ, Pontifex MB, et al. Acute exercise facilitates brain function and $\operatorname{cog}$ nition in children who need it most: an ERP study of individual differences in inhibitory control capacity. Dev Cogn Neurosci. 2014;7:53-64.

60. Labelle V, Bosquet L, Mekary S, Vu TT, Smilovitch M, Bherer L. Fitness level moderates executive control disruption during exercise regardless of age. J Sport Exerc Psychol. 2014;36(3):258-70.

61. Gates N, Fiatarone Singh MA, Sachdev PS, Valenzuela M. The effect of exercise training on cognitive function in older adults with mild cognitive impairment: a meta-analysis of randomized controlled trials. Am J Geriatr Psychiatry. 2013;21(11):1086-97.

62. Wang C, Yu JT, Wang HF, Tan CC, Meng XF, Tan L. Nonpharmacological interventions for patients with mild cognitive impairment: a meta-analysis of randomized controlled trials of cognition-based and exercise interventions. J Alzheimers Dis. 2014;42(2):663-78.

63. Zheng G, Xia R, Zhou W, Tao J, Chen L. Aerobic exercise ameliorates cognitive function in older adults with mild cognitive impairment: a systematic review and meta-analysis of randomised controlled trials. Br J Sports Med. 2016;50(23):1443-500.

64. Albinet CT, Abou-Dest A, André N, Audiffren M. Executive functions improvement following a 5-month aquaerobics program in older adults: role of cardiac vagal control in inhibition performance. Biol Psychol. 2016;115:69-77.

65. Albinet CT, Boucard G, Bouquet CA, Audiffren M. Increased heart rate variability and executive performance after aerobic training in the elderly. Eur J Appl Physiol. 2010;109(4):617-24.

66. Coetsee $\mathrm{C}$, Terblanche E. Cerebral oxygenation during cortical activation: the differential influence of three exercise training modalities. A randomized controlled trial. Eur J Appl Physiol. 2017;117(8):1617-27.

67. Doi T, Verghese J, Makizako H, Tsutsumimoto K, Hotta R, Nakakubo S, et al. Effects of cognitive leisure activity on cognition in mild cognitive impairment: results of a randomized controlled trial. J Am Med Dir Assoc. 2017;18(8):686-91.

68. Erickson KI, Voss MW, Prakash RS, Basak C, Szabo A, Chaddock L, et al. Exercise training increases size of hippocampus and improves memory. Proc Natl Acad Sci USA. 2011;108(7):3017-22.

69. Ferreira L, Tanaka K, Santos-Galduroz RF, Galduroz JC. Respiratory training as strategy to prevent cognitive decline in aging: a randomized controlled trial. Clin Interv Aging. 2015;10:593-603.

70. Gothe NP, Keswani RK, McAuley E. Yoga practice improves executive function by attenuating stress levels. Biol Psychol. 2016;121:109-16.

71. Gothe NP, Kramer AF, McAuley E. The effects of an 8-week hatha yoga intervention on executive function in older adults. J Gerontol A Biol Sci Med Sci. 2014;69(9):1109-16.

72. Gothe NP, Kramer AF, McAuley E. Hatha yoga practice improves attention and processing speed in older adults: results from an 
8-week randomized control trial. J Altern Complement Med. 2017;23(1):35-40.

73. Iuliano E, di Cagno A, Aquino G, Fiorilli G, Mignogna P, Calcagno G, et al. Effects of different types of physical activity on the cognitive functions and attention in older people: a randomized controlled study. Exp Gerontol. 2015;70:105-10.

74. Kimura K, Obuchi S, Arai T, Nagasawa H, Shiba Y, Watanabe $\mathrm{S}$, et al. The influence of short-term strength training on healthrelated quality of life and executive cognitive function. J Physiol Anthropol. 2010;29(3):95-101.

75. Klusmann V, Evers A, Schwarzer R, Schlattmann P, Reischies FM, Heuser I, et al. Complex mental and physical activity in older women and cognitive performance: a 6-month randomized controlled trial. J Gerontol A Biol Sci Med Sci. 2010;65A(6):680-8.

76. Lam LC, Chan WC, Leung T, Fung AW, Leung EM. Would older adults with mild cognitive impairment adhere to and benefit from a structured lifestyle activity intervention to enhance cognition?: a cluster randomized controlled trial. PLoS ONE. 2015;10(3):e0118173.

77. Lazarou I, Parastatidis T, Tsolaki A, Gkioka M, Karakostas A, Douka $\mathrm{S}$, et al. International ballroom dancing against neurodegeneration: a randomized controlled trial in Greek communitydwelling elders with mild cognitive impairment. Am J Alzheimers Dis Other Demen. 2017;32(8):489-99.

78. Leckie RL, Oberlin LE, Voss MW, Prakash RS, Szabo-Reed A, Chaddock-Heyman L, et al. BDNF mediates improvements in executive function following a 1-year exercise intervention. Front Hum Neurosci. 2014;8:985.

79. Liu-Ambrose T, Nagamatsu LS, Voss MW, Khan KM, Handy TC. Resistance training and functional plasticity of the aging brain: a 12-month randomized controlled trial. Neurobiol Aging. 2012;33(8):1690-8.

80. Liu-Ambrose T, Nagamatsu LS, Graf P, Beattie BL, Ashe MC, Handy TC. Resistance training and executive functions: a 12-month randomized controlled trial. Arch Intern Med. 2010;170(2):170-8.

81. Lu J, Sun M, Liang L, Feng Y, Pan X, Liu Y. Effects of momentum-based dumbbell training on cognitive function in older adults with mild cognitive impairment: a pilot randomized controlled trial. Clin Interv Aging. 2016;11:9-16.

82. Mavros Y, Gates N, Wilson GC, Jain N, Meiklejohn J, Brodaty H, et al. Mediation of cognitive function improvements by strength gains after resistance training in older adults with mild cognitive impairment: outcomes of the study of mental and resistance training. J Am Geriatr Soc. 2017;65(3):550-9.
83. Nagamatsu LS, Chan A, Davis JC, Beattie BL, Graf P, Voss MW, et al. Physical activity improves verbal and spatial memory in older adults with probable mild cognitive impairment: a 6-month randomized controlled trial. J Aging Res. 2013;2013:861893.

84. Nguyen MH, Kruse A. A randomized controlled trial of Tai chi for balance, sleep quality and cognitive performance in elderly Vietnamese. Clin Interv Aging. 2012;7:185-90.

85. Nishiguchi S, Yamada M, Tanigawa T, Sekiyama K, Kawagoe T, Suzuki M, et al. A 12-week physical and cognitive exercise program can improve cognitive function and neural efficiency in community-dwelling older adults: a randomized controlled trial. J Am Geriatr Soc. 2015;63(7):1355-63.

86. Nocera JR, McGregor KM, Hass CJ, Crosson B. Spin exercise improves semantic fluency in previously sedentary older adults. J Aging Phys Act. 2015;23(1):90-4.

87. Nouchi R, Taki Y, Takeuchi H, Sekiguchi A, Hashizume H, Nozawa $T$, et al. Four weeks of combination exercise training improved executive functions, episodic memory, and processing speed in healthy elderly people: evidence from a randomized controlled trial. Age. 2014;36(2):787-99.

88. Prehn K, Lesemann A, Krey G, Witte AV, Köbe T, Grittner U, et al. Using resting-state fMRI to assess the effect of aerobic exercise on functional connectivity of the DLPFC in older overweight adults. Brain Cogn. 2017;131:34-44.

89. Smiley-Oyen AL, Lowry KA, Francois SJ, Kohut ML, Ekkekakis P. Exercise, fitness, and neurocognitive function in older adults: the "selective improvement" and "cardiovascular fitness" hypotheses. Ann Behav Med. 2008;36(3):280-91.

90. Suzuki T, Shimada H, Makizako H, Doi T, Yoshida D, Tsutsumimoto K, et al. Effects of multicomponent exercise on cognitive function in older adults with amnestic mild cognitive impairment: a randomized controlled trial. BMC Neurol. 2012;12:128.

91. Sungkarat S, Boripuntakul S, Kumfu S, Lord SR, Chattipakorn N. Tai Chi improves cognition and plasma BDNF in older adults with mild cognitive impairment: a randomized controlled trial. Neurorehabil Neural Repair. 2018;32(2):142-9.

92. Vaughan S, Wallis M, Polit D, Steele M, Shum D, Morris N. The effects of multimodal exercise on cognitive and physical functioning and brain-derived neurotrophic factor in older women: a randomised controlled trial. Age Ageing. 2014;43(5):623-9.

93. van Uffelen JG, Chinapaw MJ, van Mechelen W, Hopman-Rock M. Walking or vitamin B for cognition in older adults with mild cognitive impairment? a randomised controlled trial. Br J Sports Med. 2008;42(5):344-51.

\section{Affiliations}

\section{Feng-Tzu Chen ${ }^{1} \cdot$ Jennifer L. Etnier ${ }^{2} \cdot$ Kuei-Hui Chan ${ }^{3} \cdot$ Ping-Kun Chiu ${ }^{3} \cdot$ Tsung-Ming Hung ${ }^{4,5} \cdot$ Yu-Kai Chang ${ }^{4,5}$}

Yu-Kai Chang

yukaichangnew@gmail.com

1 Graduate Institute of Sport, Leisure and Hospitality Management, National Taiwan Normal University, Taipei, Taiwan, ROC

2 Department of Kinesiology, University of North Carolina at Greensboro, Greensboro, NC, USA
3 Graduate Institute of Athletics and Coaching Science, National Taiwan Sport University, Taoyuan, Taiwan, ROC

4 Department of Physical Education, National Taiwan Normal University, 162, Section 1, Heping E. Rd., Taipei, Taiwan, ROC

5 Institute for Research Excellence in Learning Science, National Taiwan Normal University, Taipei, Taiwan, ROC 